\section{Aspirin for Primary Prevention of Atherosclerotic Cardiovascular Disease and Colorectal Carcinomas}

Editor - In their review of aspirin for the primary prevention of cardiovascular events, Soodi et al report aspirin's effect in the primary prevention of cardiovascular disease is unclear. ${ }^{1}$ American College of Cardiology / American Heart Association (ACC/AHA) guidelines provide the following recommendations for aspirin use for the primary prevention of atherosclerotic cardiovascular disease (ASCVD): (1) lowdose aspirin might be considered for the primary prevention of ASCVD among select adults 40 to 70 years of age who are at higher ASCVD risk but not at increased bleeding; (2) lowdose should not be administered on a routine basis for the primary prevention of ASCVD among adults $>70$ years of age; and (3) low-dose aspirin should not be administered for the primary prevention of ASCVD among adults of any age who are at increased risk of bleeding. ${ }^{2}$

As Soodi et al and the ACC/AHA advise, the clinician must balance an understanding of a patient's estimated ASCVD risk with potential benefits and adverse risk from pharmacological therapy in the context of a risk discussion. In addition to reviewing the potential benefit of aspirin for the primary prevention of ASCVD, we believe the risk discussion should also note the potential benefit of long-term aspirin use in reducing the risk of developing overall cancer and colorectal cancer. In an analysis of over 135,000 participants, the use of aspirin for 6 years or longer was associated with a $19 \%$ decreased risk of colorectal cancer and a $15 \%$ decreased risk of any type of gastrointestinal cancer. ${ }^{3}$ Investigators concluded long-term aspirin use was associated with modest but significantly reduced risk of overall cancer, especially gastrointestinal tumors. The United States Preventive Services Task Force recommends initiating low-dose aspirin use for the primary prevention of cardiovascular disease (CVD) and colorectal cancer in adults aged 50 to 59 years who have a $10 \%$ or greater 10-year CVD risk, are not at increased risk for bleeding, have a life expectancy of at least 10 years, and are willing to take low-dose aspirin daily for at least 10 years. ${ }^{4}$

In sum, we agree with Soodi et al that a thoughtful discussion between patients and their doctors should be conducted before beginning aspirin for primary prevention of cardiovascular events. When those discussions occur, the potential role of longterm aspirin use in preventing the development of colorectal cancer should also be reviewed so that patients will be fully informed of the potential benefits of long-term aspirin use.

Keywords: Cardiovascular; Aspirin; Colorectal carcinomas; Primary prevention

\section{References}

1. Soodi D, VanWormer JJ, Rezkalla SH. Aspirin in Primary Prevention of Cardiovascular Events. Clin Med Res. 2020;18 (2-3):89-94.

2. Arnett DK, Blumenthal RS, Albert MA, et al. 2019 ACC/ AHA Guideline on the Primary Prevention of Cardiovascular Disease: A Report of the American College of Cardiology/American Heart Association Task Force on Clinical Practice Guidelines [published correction appears in J Am Coll Cardiol. 2019 Sep 10;74(10):1429-1430] [published correction appears in J Am Coll Cardiol. 2020 Feb 25;75(7):840]. J Am Coll Cardiol. 2019;74(10):e177-e232.

3. Cao Y, Nishihara R, Wu K, et al. Population-wide Impact of Long-term Use of Aspirin and the Risk for Cancer [published correction appears in JAMA Oncol. 2019 Apr 1;5(4):579]. JAMA Oncol. 2016;2(6):762-769.

4. Aspirin Use for the Primary Prevention of Cardiovascular Disease and Colorectal Cancer: Recommendation Statement. Am Fam Physician. 2016;94(8):Online.

\section{Author Affiliations}

Anna Breiburg, APRN-BC, RRT*; and Ware G. Kuschner, MD†

*Pulmonary Section, Veterans Affairs Palo Alto Health Care System, Palo Alto, California, USA; Email: anna.breiburg@, va.gov

†Pulmonary Section, Veterans Affairs Palo Alto Health Care System, and Division of Pulmonary, Allergy \& Critical Care Medicine, Department of Medicine, Stanford University School of Medicine, Palo Alto, California, USA; Email: ware.kuschner@ va.gov

Received: October 8, 2020

Accepted: November 20, 2020

doi: $10.3121 / \mathrm{cmr} \cdot 2020.1633$

\section{Author Response to "Aspirin for Primary Prevention of Atherosclerotic Cardiovascular Disease and Colorectal Carcinomas"}

Editor - We appreciate your interest in our article published in Clinical Medicine \& Research. ${ }^{1}$ Since the guidelines were published in a cardiology journal, we felt it would be beneficial to clarify and discuss the recommendations in an internal medicine journal. Primary care providers are the front lines to guide their patients regarding aspirin use for primary prevention. Our discussion started from the studies published 
in the early 1980's for secondary prevention and ended with the most recent primary prevention studies. The conclusions hopefully provide a clear guide to clinicians to be able to provide the best care for their patients. The article focuses on aspirin use for cardiovascular disease only. Thank you again for your interest.

Keywords: Cardiovascular; Aspirin; Colorectal carcinomas; Primary prevention

\section{References}

1. Soodi D, VanWormer JJ, Rezkalla SH. Aspirin in primary prevention of cardiovascular events. Clin Med Res. 2020;18 (2-3):89-94.

\section{Author Affiliations}

Deepa Soodi, MD*; Jeffrey J. VanWormer, PhD'; and Shereif H. Rezkalla, MD, FACP, FACC

*Department of Internal Medicine, Marshfield Clinic Health System, Marshfield,WI; Email: soodi.deepa@

marshfieldclinic.org

Center for Clinical Epidemiology \& Population Health, Marshfield Clinic Research Institute, Marshfield, WI; Email: vanwormer.jeffrey@marshfieldresearch.org

${ }^{*}$ Department of Cardiology, Marshfield Clinic Health System, Marshfield, WI; Email: Rezkalla.shereif@,

marshfieldclinic.org

Received: November 18, 2020

Accepted: December 9, 2020

doi: $|0.3| 2 \mid / \mathrm{cmr} .2020 .1645$ 